\title{
IMAGE PROCESSING FOR MEDICAL EVALUATION ON NBI ENDOSCOPY IMAGES
}

Vítor Gabriel Sgobbi Martins Silva, Elcio H. Shiguemori, Wellington Cardia.

UNIP - Universidade Paulista, São José dos Campos, SP, Brazil

IEAv - Institute for Advanced Studies, São José dos Campos, SP, Brazil

\begin{abstract}
The employment of digital images has grown in recent years in several areas. Mainly due by technological and computer development, its use has a larger amount of available information in applications such as: security, navigation and medicine. In most applications, the use of image processing techniques for automatic information extraction has been needed to help experts and professionals in several areas. At this paper is presented an application of image processing and computer vision techniques with images obtained by the new NBI (Narrow Band Imaging) equipment, an endoscopic technique that has been used to enhance the accuracy of diagnosis by using narrow-bandwidth filters in a red-green-blue (R/G/B) sequential illumination system. Several techniques for image processing have been employed in this work to highlight some lesions in order to assist diagnosis of flat lesions found in intestines or stomach.
\end{abstract}

Keywords: NBI Endoscopy, Image Processing, Medical Technology

\section{INTRODUCTION}

The application of image processing has grown on different areas and it has been improved for human and machine interpretation. Since 1964 the area of Image Processing has been applied in almost all human activities (Russ, 2011). In medicine, the image processing has been used for medical diagnosis and it has become usual mainly due to advances computational interpretation and the development of new sensors and equipment. Digital image processing involves procedures that can usually be implemented via software or dedicated hardware. The use of specialized hardware for image processing has been common due to technological development, with the use of graphics cards (Marques Filho, Vieira Neto, 1999).

This article aims to apply techniques of image processing and computer vision to extract information from images NBI. Even in conventional imaging or NBI, some details are not easily seen without the processing of these images. With this processing, some deficiencies 
can be highlighted to aid in medical diagnosis. The contribution of this study is conducted for the particular type of new technology system called NBI endoscopy, cooperating with its use and development.

Mizuno et al. (2003) have analyzed a hypoglottis (ranula) image, they confirmed that the blue image shows the microblood vessels network located near the surface mucosa and the green image deeply shows the blood vessels. With an adenomatous polyp image they concluded that the NBI images were more appropriated than the conventional magnifying endoscopy and more staining.

For Gheorghe (2006), the Barrett's esophagus analysis depends on random biopsies for detection of intestinal metaplasia (cell alteration). Using NBI technology the metaplasia area can be identified for biopsy. The time and cost can be minimized by obtaining specified tissue areas for biopsy instead obtaining multiple areas in random biopsies. Also according to Gheorghe (2006), tumors can represent some cancer mortality; these tumors can be located at stomach, esophagus and large bowel.

A kind of cancer prevention is the early detection when a lesion is at the stage that is still curable. The NBI magnifying endoscopy is more effective for detecting premalignant and malignant precursors, advanced which is in tumor process (Gheorghe, 2006).

\section{NBI IMAGES}

Images obtained by the NBI filter technology system offer higher definition, sharpness, contrast and color quality (Olympus, 2011); these colors provides high blue tracks decreasing the red light allowing better light absorption of mucosa surface. NBI is based on use of optical filters to isolate two specific bands of light: Blue $(415 \mathrm{~nm})$ and Green $(540 \mathrm{~nm})$, which replaces the RGB broadband with a filter of conventional light source, which uses all the spectrum of visible light (400 nm and $700 \mathrm{~nm}$ ) (Olympus, 2011).

\section{NBI IMAGE PROCESSING}

The red band of hue $(625-740 \mathrm{~nm})$, as orange $(590-625 \mathrm{~nm})$ and yellow $(565-590 \mathrm{~nm})$ can be visualized as anomalies tissue located in different regions as esophagus, stomach, colon and others (Bovik, 2009).

In this work, to assist the evaluation diagnosis on NBI endoscopy images, the first procedure applied on transforming the NBI image color system from RGB (Red, Green, Blue) to HSV (Hue, Saturation e Brightness) and then generates three new images. This conversion is commonly used on image processing to facilitate the computational and human interpretation (Russ, 2011). Hue can be recognized in HSV color space, it represents the primary color; in this case, red is meant to be highlighted, in general aspects to identify anomalies like ulcers, infections and inflammations. The NBI filter has an intense aspect of green and blue band; red tissue can therefore be characterized as an abnormal tissue.

Equation 3.1 shows the saturation channel; its value can be calculated by using the conversion for each RGB red-green-blue band value for HSV (Russ, 2011): 


$$
S=1-\frac{3}{R+G+B} \operatorname{hin} R, G, B_{=}^{-}
$$

In this paper, a "HS Filter" is applied to segment the minimum and max values of Hue and Saturation channels. The filtering is applied only on the region of interest of Saturation resulting image. This procedure aims to return the image highlighting primary differences between anomalies aspects that often have predominant colors between red and yellow. At this procedure it was necessary to apply different techniques and algorithms. Some parameters were obtained by testing different endoscopic images and finding specifics values for its parameters assigned to a mask image, these parameters were specified for only images used for study that have same aspects. For visualization, the last step was apply a subtraction in the resulting image from the original, to return only regions of interesting, in this case, the expected anomaly. The subtraction is made pixel by pixel from the original, shown in Equation 3.2 , in this operation there are ranges of possible values for images, which the first range is 0 to 255 becomes -255 to 255 (Bovik, 2009). The data can be resized to fit on a single byte, then replacing the original image.

$$
B=\operatorname{int} \cdot \frac{(\text { Sum }- \text { Min })}{(\text { Max }- \text { Min })}
$$

where $B$ is the background image, int is the memory capacity of the image, usually 255. Sum, Max and Min refer to pixel values.

\section{RESULTS}

At this study we used three images available on NBI endoscopy Gastrolab (2012). The images were displayed at "Gastrolab Image Library and Endoscopic Archives" and can freely be used for noncommercial purpuses (Gastrolab, 2012).

For the HS Filter, the Hue values employed were 0 to 180 and Saturation 0 to 255. Figure 4.1 represents a Barrett's esophagus, their cause is related to a metaplasia; cell change caused by the chronic adaptation to exposure to the acid reflux esophagitis (Barrett, 1957). The Figure 4.1 (b) shows the saturation channel captured with image processing.

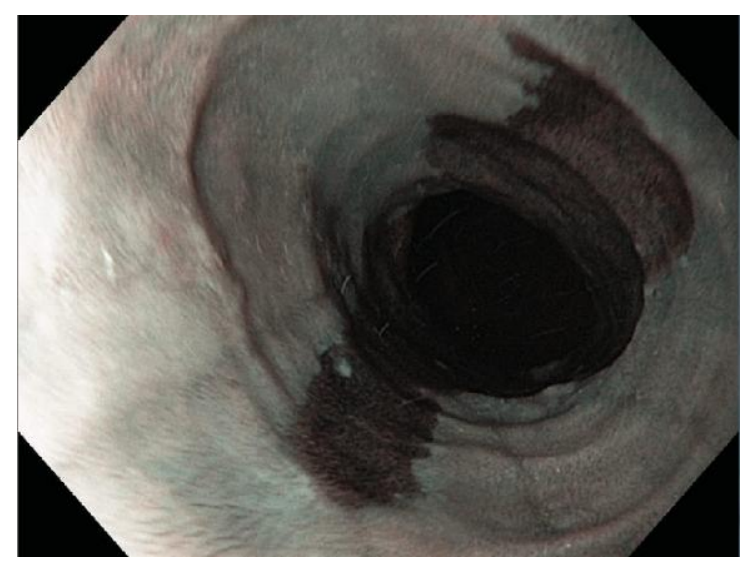

(a) 


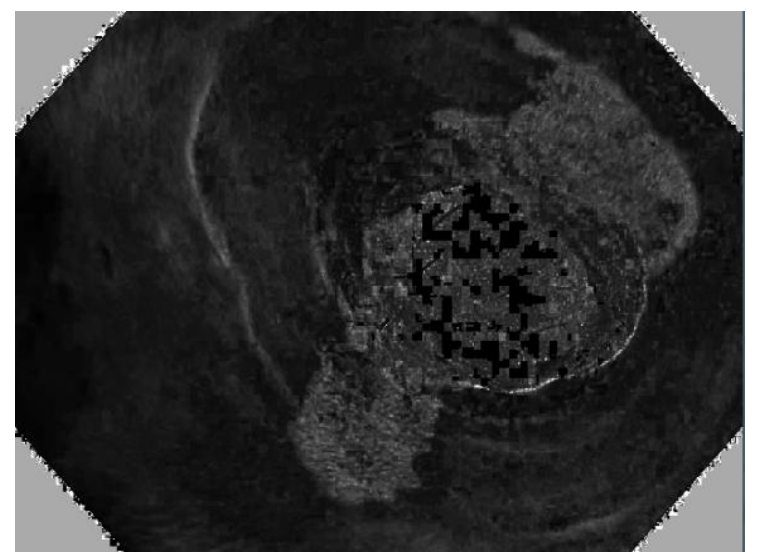

(b)

Figure 4.1 - Barret's esophagus. (a) Original NBI image; (b) Saturation Channel of HSV.

Figure 4.2 (b) shows a Peptic Ulcer highlighted with the HS Filter where it's possible to see a small portion at the top where the epithelial tissue is also uncommon.

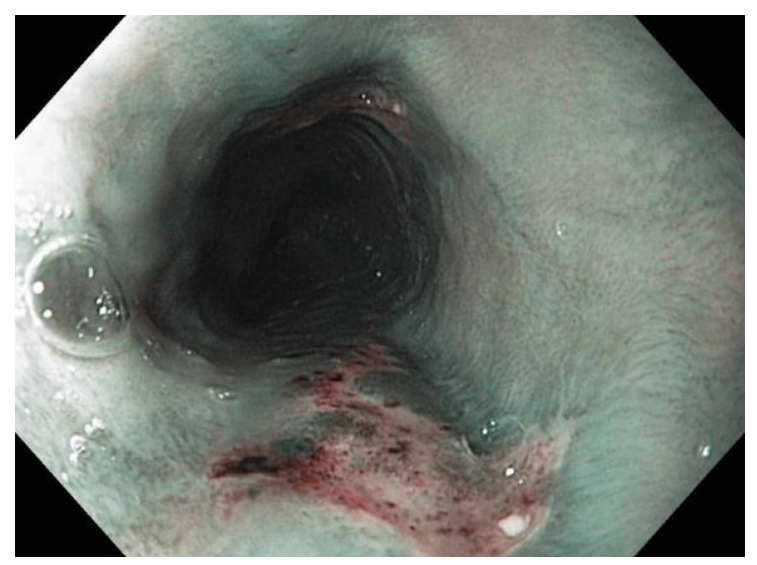

(a)

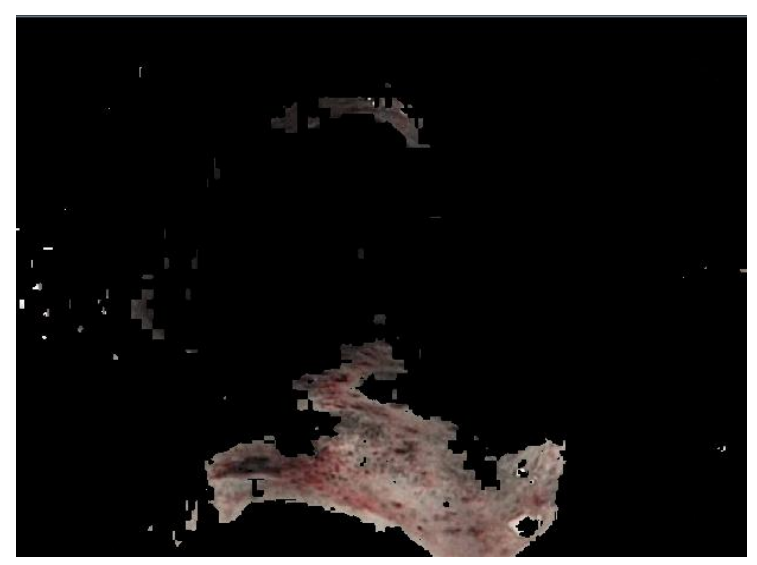

(b)

Figure 4.2 - Peptic Ulcer. (a) Original NBI image; (b) Processed image.

Source: Gastrolab, 2010. 
Figure 4.3 represents another Peptic Ulcer NBI image, located on top and left part of image. By using "HS Filter" for the pattern extraction, there is a large amount of abnormal tissue extracted.

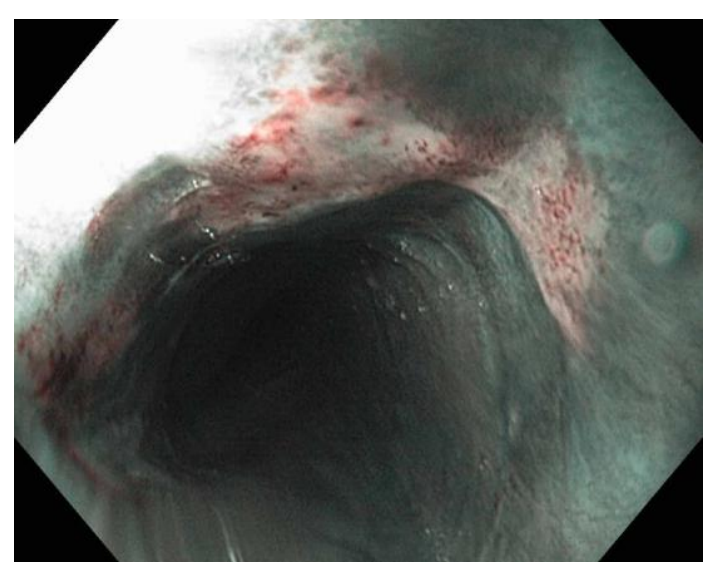

(a)

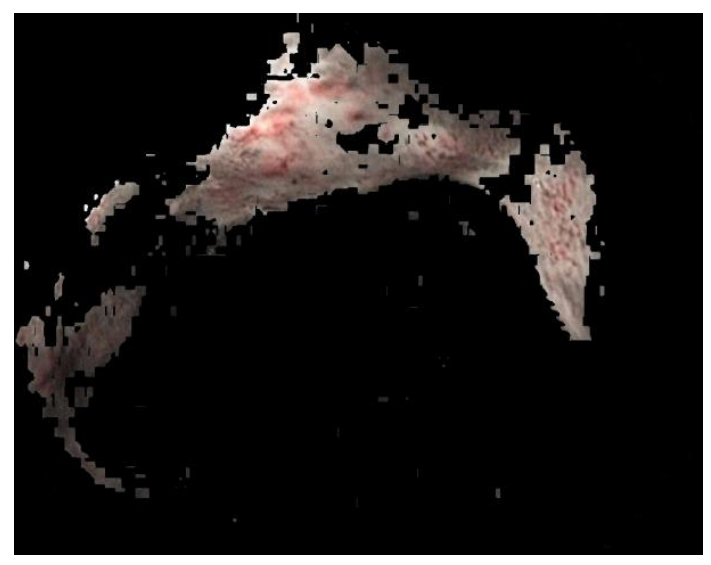

(b)

Figure 4.3 - Other Peptic Ulcer. (a) Original NBI image; (b) Processed image.

Source: Gastrolab, 2010.

\section{FINAL REMARKS}

With this work were presented some results obtained with the image processing and computer vision on images NBI endoscopy. Some techniques have been applied to the application. With "HS Filter" was possible to highlight some anomalies with aspects of disease.

The methodology can be applied to other diseases, with small variations of parameters. This study is still under development and it is expected to implement new methods. Time and computational costs can be minimized aiming to help professionals obtain diagnosis. Random biopsies could be also minimized with images obtained by NBI filter technology. 


\section{REFERENCES}

[1] BARRETT, N. The lower esophagus lined by columnar epithelium. Surgery 41 (6): 881-94, 1957.

[2] BOVIK, A. C. The Essential Guide to Image Processing. Elsevier Inc, 2009.

[3] CANNY, J. Finding edges and lines in images. M.S. thesis, M.I.T. Cambridge, MA, USA, 1983.

[4] GASTROLAB, Project 365: An Endoscopy Pic Every Day of the Year. October - December 2010. Available on: <http://www.kolumbus.fi/hans/gastrolab/pre10-4.htm>. Access: January, $20^{\text {th }}, 2012$.

[5] GHEORGHE, C. Narrow-Band Imaging Endoscopy for Diagnosis of Malignant and Premalignant Gastrointestinal Lesions, Center of Gastroenterology and Hepatology, Fundeni Clinical Institute, Bucharest 2006.

[6] INTEL, Open Source Computer Vision Library: reference manual, 2001.

[7] MARQUES FILHO, OGÊ; VIEIRA NETO, HUGO. Processamento Digital de Imagens. Rio de Janeiro: Brasport, 1999.

[8] MIZUNO H, GONO, K, TAKEHANA S, et al. Narrow band imaging technique. Techn Gastrointest Endosc 2003; 5: 78-81

[9] OLYMPUS, NBI In Endoscopy. Available on: <http://www.olympuseuropa.com/endoscopy/425_7552.htm\#7555>. Access: December, $8^{\text {th }}, 2011$.

[10] OLYMPUS, Narrow Band Image (NBI). Available on: < http://www.olympuslatinoamerica.com/portuguese/msg/msg_nbi_port.asp?d=9\&s=36\&c= 72>. Access: December, $8^{\text {th }}, 2011$.

[11] RUSS, J. C. The Image Processing Handbook, Sixth Edition. Boca Raton, FL: CRC PRESS, 2011. 